\title{
Perfil e conduta dos cirurgiões-dentistas de Patos-PB frente aos traumatismos dentoalveolares
}

\author{
Profile and conduct of dental surgeons of the public health system of Patos-PB face to dental trauma \\ Perfil y conducta de los odontólogos del sistema público de salud de Patos-PB frente a los traumatismos dentales \\ Faldryene de Sousa QUEIROZ ${ }^{1}$ \\ UFCG - Universidade Federal de Campina Grande, 58708-110 Patos-PB, Brasil
}

\section{Resumo}

Os traumatismos dentários são frequentes na rotina clínica dos Cirurgiões-Dentistas e a correta conduta do tratamento é fundamental para um bom prognóstico. Objetivo: O objetivo deste trabalho foi avaliar o perfil e a conduta dos cirurgiões-dentistas das 41 Unidades Básicas de Saúde do município de Patos-PB frente aos traumatismos dentários. Metodologia: Para coleta de dados foi aplicado um questionário contendo perguntas sobre o perfil demográfico dos profissionais e a experiência com traumatismo dental. Os dados foram analisados estatisticamente pelo Qui-quadrado, com margem de erro de 5\%. Resultados: 66,7\% dos Cirurgiões-dentistas eram do sexo feminino, 83,3\% fizeram a graduação em instituição de ensino pública, 33,3\% possuíam mais de 10 anos de formados, 53,3\% eram especialistas, apenas 10\% relataram ter algum tipo de cursos na área e a especialidade predominante foi a de Saúde da Família (16,7\%). Sobre a prevalência do trauma dental na rotina de atendimentos, 46,7\% dos entrevistados relataram ter recebido pacientes com traumatismo dentário, destes, 36,7\% receberam apenas casos de fratura dental e $10 \%$ receberam tanto fratura como avulsão dental, 92,8\% realizaram o primeiro atendimento e $7,2 \%$ encaminharam para outro local e 56,7\% relataram sentirem-se seguros para realizar o atendimento. A relação entre a segurança no atendimento com variáveis de perfil e conduta não demonstrou associação estatisticamente significante (p>0,05). Conclusão: Observou-se uma alta prevalência de traumatismo dentário e que muitos profissionais ainda não se sentem seguros para prestarem o atendimento imediato, sendo necessário mais incentivo em cursos na área, visando otimizar a assistência ao paciente com trauma dental.

Descritores: Odontólogos; Conhecimento; Traumatismo Dentário.

\section{Abstract}

Dental traumatisms were a relatively frequent situation in the daily routine of the Dental Surgeon and their correct conduct is fundamental for a good prognosis of the treatment. Objective: The objective of this study was to evaluate the profile and conduct of dental surgeons of the 41 Basic Health Units of the city of Patos-PB in the face of dental trauma. Methodology: A questionnaire containing questions about the demographic data of the professionals and the experience about dental traumatism was used to collect data.The data were analyzed statistically by chi-square, with a margin of error of 5\%. Results: $66.7 \%$ of dental surgeons were female, $83.3 \%$ graduated from a public educational institution, $33.3 \%$ had more than 10 years of training, $53.3 \%$ of professionals were specialists, only $10 \%$ reported having some type of courses in the area and the predominant specialty was Family Health (16.7\%). 46.7\% of the interviewees reported receiving dental trauma patients, of whom $36.7 \%$ received only cases of dental fracture and $10 \%$ received both fracture and dental avulsion, $92.8 \%$ performed the first care and $7.2 \%$ sent to another location and $56.7 \%$ reported feeling able to perform care. The relationship between the ability to attend and variables of profile and conduct didn't show a statistically significant association ( $\mathrm{p}>0.05)$. Conclusion: It was observed a high prevalence of dental traumatism and that many professionals still do not feel safe to provide the care, being necessary more incentive in courses in the area, aiming to optimize the dental patient care.

Descriptors: Dentists; Knowledge; Tooth Injuries.

\section{Resumen}

Los traumatismos dentales son frecuentes en el cotidiano clínico de los Cirujanos-Dentistas y su correcta conducta es fundamental para un buen pronóstico del tratamiento. El objetivo del trabajo fue evaluar el perfil y conducta de los cirujanos-dentistas del sistema público de Patos-PB a los traumatismos dentales. Para la recolección de datos se aplicó un cuestionario que contenía preguntas sobre el perfil demográfico de los profesionales y la experiencia con traumatismo dental. Los datos fueron analizados estadísticamente por el Qui-cuadrado, con margen de error del 5\%. 66,7\% de los Cirujanos-dentistas eran del sexo femenino, 83,3\% hicieron la graduación en institución pública, $33,3 \%$ poseía más de 10 años de graduados, 53,3\% de los profesionales eran especialistas, apenas 10\% relataron tener algún tipo de cursos en el área y la especialidad predominante fue la de Salud de la Familia (16,7\%). En cuanto a la prevalencia del trauma dental en la rutina, 46,7\% de los entrevistados relataron haber recibido pacientes con traumatismo dental, de éstos, $36,7 \%$ recibieron sólo casos de fractura dental y el $10 \%$ recibieron tanto fractura como avulsión dental, 92,8 \% realizaron la primera atención y 7,2\% encaminó a otro local y 56,7\% relató sentirse seguros para realizar la atención. La relación entre la seguridad en la atención con variables de perfil y conducta no demostró asociación estadísticamente significativa ( $>$ > 0,05). Se observó una alta prevalencia de traumatismo y que muchos profesionales aún no se sienten seguros para la atención, necesitando más incentivo en cursos del área, buscando optimizar la atención.

Descriptores: Odontólogos; Conocimiento; Traumatismos de los Dientes.

\section{INTRODUÇÃO}

O traumatismo dentário é uma agressão térmica, química ou mecânica sofrida pelo dente e estruturas adjacentes, cuja magnitude supera a resistência encontrada nos tecidos ósseos e dentários e a sua extensão tem relação direta com a intensidade, tipo e duração do impacto ${ }^{1}$. Segundo Piva et al. ${ }^{2}$ as arcadas dentárias por se localizarem numa projeção corporal anterior ficam assim mais expostas às agressões externas, aumentando a propensão à esse tipo de lesão.

Os traumatismos dentários podem ocorrer de forma frequente na população de diferentes faixas etárias, com alta prevalência em crianças e adolescentes, trazendo diversas consequências aos dentes e estruturas adjacentes. A correta realização dos primeiros socorros pós-trauma é de extrema importância, pois o prognóstico está diretamente relacionado à conduta clínica do cirurgião-dentista ${ }^{3}$.

$\mathrm{O}$ alto índice de violência e acidentes automobilísticos e um maior número de crianças participando em esportes radicais atuam como contribuintes para o aumento da prevalência dos 
traumatismos dentais ${ }^{4}$. Estima-se que, em um futuro próximo, a prevalência de traumatismos dentoalveolares poderá exceder a de doenças como a cárie e doenças periodontais, sendo hoje já considerado um importante problema de saúde pública entre crianças e adolescentes 5 .

Ocorrem mais em crianças do sexo masculino com idade de cerca de três anos e meio ${ }^{6,7}$. Na dentição permanente, há maior incidência nas idades entre 9 e 10 anos para os meninos ${ }^{8}$ e em relação ao sexo masculino foi predominante segundo os estudos de Torres Silva et al. ${ }^{9}$, sendo o incisivo central superior o dente mais acometido $^{10}$.

Há vários tipos de traumatismos, desde uma simples trinca de esmalte, onde não há perda do material dental, até a avulsão completa do dente, onde ele é removido por completo da boca. Nestes casos, o tempo decorrido, o condicionamento do dente e a correta técnica do cirurgião-dentista são cruciais para que haja sucesso nos casos em que se opta pelo reimplante ${ }^{11}$.

Como consequências dos traumatismos dentários, a necrose pulpar mostra-se como a mais frequente $^{12}$, bem como os impactos sobre as vítimas, como danos estéticos, psicológicos e sociais, além de produzir significativos custos ${ }^{13}$.

Kremer et al. ${ }^{3}$ afirmam que os traumatismos dentários constituem uma situação relativamente frequente no cotidiano dos atendimentos clínicos efetuados pelo cirurgião-dentista. São situações de urgência odontológica que impõem ao profissional um atendimento rápido, porém minucioso. Apesar da agilidade no primeiro atendimento, na maioria das vezes é necessário o acompanhamento do paciente por um longo período.

Portanto, segundo Pinheiro e Delfino ${ }^{14}$ a correta conduta do cirurgião-dentista na assistência à vítima de trauma dentária, baseada em seu treinamento formal adquirido na graduação, as experiência clínicas e o conhecimento adquiridos são fundamentais na escolha de opções de tratamento, melhorando os resultados finais. A procura imediata por tratamento especializado é fundamental para o diagnóstico e adoção de um correto procedimento, a fim de se obterem bons prognósticos.

Diante do exposto, o presente trabalho se propõe a avaliar o perfil, conhecimento e conduta dos cirurgiões-dentistas das Unidades Básicas de Saúde da cidade de Patos-PB frente aos traumatismos dentoalveolares.

\section{MATERIAL E MÉTODO}

Este trabalho foi aprovado pelo Comitê de Ética em Pesquisa com Seres Humanos das Faculdades Integradas de Patos - FIP (Patos-PB, Brasil, sob parecer $\mathrm{n}^{\mathrm{o}} 2.872 .175$.

O estudo foi do tipo observacional, desenvolvido por meio da aplicação de um questionário, aplicado no período de março a setembro de 2018, para a avaliação da experiência e conduta dos CirurgiõesDentistas, atuantes na atenção básica, frente aos traumatismos dentoalveolares.

A cidade de Patos-PB é assistida por 41 Unidades Básicas de Saúde da Família (USF), com um cirurgião-dentista (CD) para cada USF. O universo foi composto por todos os CD's que trabalham no sistema público de saúde, especificamente nas Unidades Básicas de Saúde, registrados no CRO/PB e encontrados em seus respectivos locais de trabalho.

Foram incluídos na pesquisa todos os CDs que trabalhavam nas Unidades Básicas de Saúde (UBS) da cidade de Patos - PB e excluídos os que se negaram a responder o questionário, que não assinaram o Termo de Consentimento Livre e Esclarecido (TCLE) ou que não responderam em tempo aos questionários; profissionais que assumiram o emprego após a data inicial da pesquisa e os profissionais que trabalhavam em mais de um estabelecimento público na cidade onde a pesquisa foi desenvolvida.

Para a realização da pesquisa, foi utilizado um questionário autoaplicado, fechado e estruturado, respondido pelo pesquisador, contendo um total de 20 questões sendo 10 sobre os dados referentes à identificação do sujeito da pesquisa e à formação profissional e 10 questões específicas sobre o conhecimento dos mesmos e suas condutas adotadas frente aos diversos tipos de traumatismos dentoalveolares, como fraturas com envolvimento pulpar, fraturas radiculares e avulsões. Estas questões foram extraídas de questionários validados descritos nos estudos de Pinheiro e Delfino ${ }^{14}$, Granville-Garcia et al. $^{15}$, Costa et al. ${ }^{16}$ e Hu et al. ${ }^{17}$.

Para a aplicação do questionário o pesquisador visitou pessoalmente todos os CDs, os quais foram previamente comunicados sobre os propósitos dessa pesquisa e do caráter voluntário e anônimo. Os questionários foram entregues para preenchimento, em envelope fornecido pelo pesquisador, contendo o TCLE para que fossem respondidos e foram recebidos em até duas semanas após a data da entrega, sendo essa determinação previamente comunicada aos participantes.

As respostas dos CDs foram analisadas através de procedimentos de estatística descritiva, realizados por meio do programa SPSS $®$ (Statistical Package for the Social Sciences) versão 20.0 para Microsoft Windows, com teste de associações pela análise do Quiquadrado entre algumas variáveis da caracterização da amostra e resultados das condutas dos CDs em casos de traumatismos dentoalveolares.

Os resultados foram expressos em números absolutos e percentuais, apresentados através de Gráficos e Tabelas.

\section{RESULTADOS}

- Perfil dos cirurgiões-dentistas das Unidades Básicas de Saúde de Patos-PB

A pesquisa teve como universo os Cirurgiõesdentistas das 41 Unidades Básicas de Saúde do Município de Patos-PB e houve uma perda amostral de 11 CDs, pelos seguintes critérios: 2,43\% por encontrarem-se de licença para tratamento de saúde; 
$2,43 \%$ de licença maternidade; $2,43 \%$ por não responderem ao questionário à tempo; $4,87 \%$ por recusaram-se a responder o questionário e $14,6 \%$ por não se encontrarem no local de trabalho no momento de aplicação do questionário; resultando assim uma amostra final de 30 Cirurgiões-dentistas que responderam ao questionário aplicado pelo pesquisador.

Quanto ao perfil dos entrevistados, 66,7\% $(n=20)$ eram do sexo feminino, com idade variando de 22 a 62 anos, e média de 35,6 anos. Sobre a formação profissional, a maioria $(83,3 \%, \quad n=25)$ possuía graduação em Instituição de Ensino Pública e, referente aos anos de formação, 16,7\% (n=5) possuíam menos de 1 ano de formados enquanto que 33,3\% (n=10) possuíam mais de 10 anos de formados. Quanto à Pósgraduação 53,3\% $(n=16)$ dos CD's eram especialistas e apenas $10 \%(\mathrm{n}=3)$ relataram possuir algum tipo de cursos em Emergência de Lesões Traumáticas.

Com relação à distribuição dos cirurgiõesdentistas por especialidades, pôde-se observar que a especialização em Saúde da Família foi a mais prevalente $(16,7 \%)$, seguido da endodontia (10\%). As especialidades de Odontopediatria, Dentística, Odontologia do Trabalho, Implatodontia e Ortodontia, representaram, cada uma, 3,3\% da amostra, 6,7\% relatam ter duas especialidades e 3,3\% mais de duas.

A atuação apenas no Sistema Público de Saúde correspondeu a $70 \%(\mathrm{n}=21)$, enquanto que $30 \%(\mathrm{n}=9)$ atuavam tanto no sistema público como no privado.

- Experiência e conduta frente aos traumatismos dentais

Sobre os dados referentes à prevalência do trauma dental na rotina de atendimentos, 46,7\% $(\mathrm{n}=14)$ dos CDs relataram ter recebido pacientes com traumatismos dentários. Com relação ao tipo de trauma, $53,3 \% \quad(\mathrm{n}=16) \quad$ nunca receberam, 36,7\% $\quad(\mathrm{n}=11)$ receberam apenas casos de fratura dental e $10 \%(\mathrm{n}=3)$ relataram ter recebido tanto fratura como avulsão dental. Dos que receberam pacientes com trauma, 92,8\% ( $\mathrm{n}=13)$ realizaram o primeiro atendimento e $7,2 \%(\mathrm{n}=1)$ encaminharam para outro local. Quanto à segurança para prestação de atendimento aos pacientes com casos de traumatismos dentários, levando em conta tanto os que já receberam pacientes ou nunca receberam, a maioria 56,7\% (n=17) relatou sentir-se seguros para realizar o atendimento (Gráfico 1).

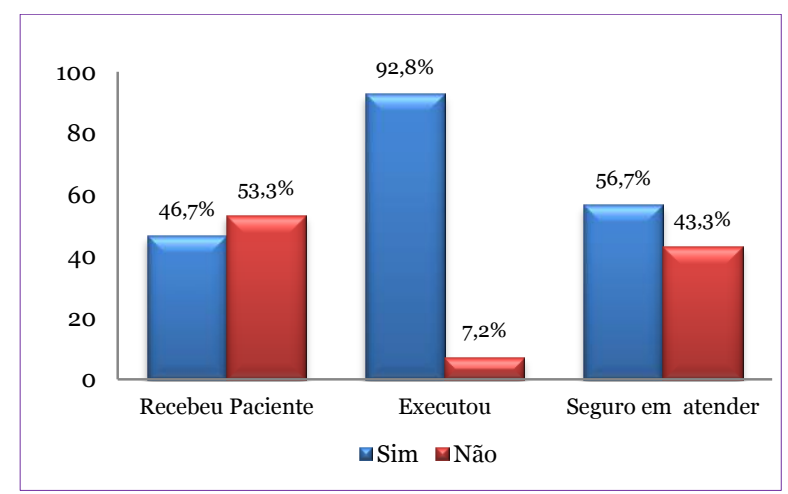

Gráfico 1. Prevalência do traumatismo dental e conduta em relação ao atendimento

\section{- Análise das respostas relativas aos casos clínicos propostos}

Em relação às 10 questões específicas sobre a conduta nos casos de traumatismo dentário, observou-se que nenhum cirurgião-dentista acertou todas as questões, 36,7\% ( $n=11)$ erraram 04 ou mais questões, $33,3 \%(n=10)$ erraram 03 questões, 20\% $(n=6)$ erraram 02 e $10 \%(n=3)$ erraram apenas uma questão dos casos propostos.

O Gráfico 2 demonstra o percentual de acertos e erros por questões propostas nos casos clínicos da segunda parte do questionário.

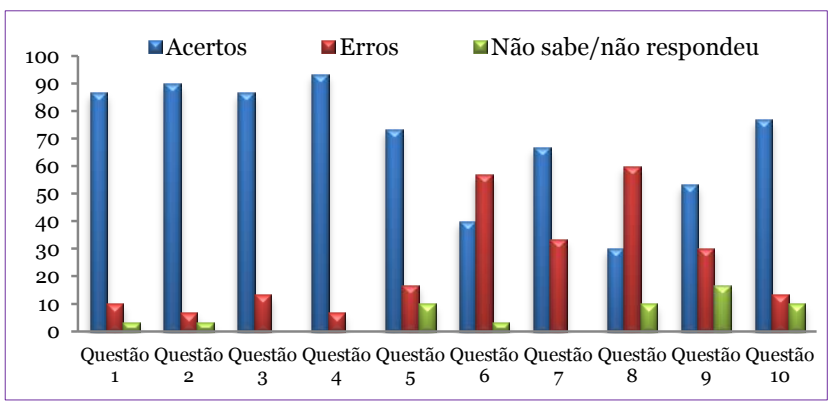

Gráfico 2. Avaliação das respostas aos casos clínicos propostos

\section{- Análise Bivariada}

A Tabela 1 apresenta a relação entre a segurança em atender os pacientes com trauma dental e o perfil e conduta dos profissionais.

Tabela 1. Relação entre a segurança no atendimento e o perfil e conduta dos cirurgiões-dentistas

\begin{tabular}{|c|c|c|c|c|}
\hline \multicolumn{5}{|c|}{ Segurança em atender paciente com trauma } \\
\hline & Sim & Não & Total & Valor de $\mathrm{p}$ \\
\hline Variáveis CD & $\mathrm{n}(\%)$ & $\mathrm{n}(\%)$ & $\mathrm{n}(\%)$ & \\
\hline \multicolumn{5}{|l|}{ Sexo } \\
\hline Masculino & $7(70,0)$ & $3(30,0)$ & $10(100,0)$ & \multirow{3}{*}{0,297} \\
\hline Feminino & $10(50)$ & $10(50)$ & $20(100,0)$ & \\
\hline \multicolumn{4}{|l|}{ Idade } & \\
\hline 20-30 anos & $8(53,3)$ & $7(43,7)$ & $15(100,0)$ & \multirow{3}{*}{0,426} \\
\hline $31-40$ anos & $6(75,0)$ & $2(25,0)$ & $8(100,0)$ & \\
\hline$>40$ anos & $3(42,9)$ & $4(57,1)$ & $7(100,0)$ & \\
\hline \multicolumn{5}{|l|}{ Anos de Formado } \\
\hline Até 5 anos & $8(57,1)$ & $6(46,9)$ & $14(100,0)$ & \multirow{2}{*}{0,967} \\
\hline$>5$ anos & $9(56,2)$ & $7(43,8)$ & $16(100,0)$ & \\
\hline \multicolumn{5}{|l|}{ Pós-graduação } \\
\hline Sim & $9(56,2)$ & $7(43,8)$ & $16(100,0)$ & \multirow{2}{*}{0,961} \\
\hline Não & $8(57,1)$ & $6(42,9)$ & $14(100,0)$ & \\
\hline \multicolumn{5}{|l|}{ Local de trabalho } \\
\hline Pública & $10(47,6)$ & $11(52,4)$ & $21(100,0)$ & \multirow[t]{2}{*}{0,127} \\
\hline Pública e Particular & $7(77,8)$ & $2(22,2)$ & $9(100,0)$ & \\
\hline \multicolumn{5}{|c|}{ Conduta frente ao trauma } \\
\hline Nunca recebeu & $11(68,8)$ & $5(31,2)$ & $16(100,0)$ & \multirow[t]{3}{*}{0,241} \\
\hline Executou atendimento & $6(46,2)$ & $7(53,8)$ & $13(100,0)$ & \\
\hline Encaminhou & $0(0,0)$ & $1(100,0)$ & $1(100,0)$ & \\
\hline
\end{tabular}

\section{DISCUSSÃO}

O perfil dos profissionais entrevistados na presente pesquisa se assemelha com os de Pinheiro e Delfino $^{14}$ onde $60,78 \%$ eram do sexo feminino e possuíam média de 36 anos de idade, também se assemelham aos dos estudos de Leôncio et al. ${ }^{18}$ onde $52 \%$ eram do sexo feminino e encontraram a média de idade de 20-30 anos, porém este mesmo estudo mostra os anos de graduação entre 1 a 5 representando $34 \%$ e a predominância de $60 \%$ atuando em instituição pública e privada o que difere dos resultados desta pesquisa em questão onde os profissionais com mais de 10 anos de profissão representam $33,3 \%$ e a atuação em instituição exclusivamente pública predomina com percentual de $70 \%$. 
O fato da maioria dos CD's serem do gênero feminino e com tempo de formação profissional com mais de 10 anos corrobora com o estudo de Antunes et al. ${ }^{19}$. Mas o gênero feminino predominante discorda do estudo de França et al. ${ }^{20}$ que entrevistaram uma maior quantidade de homens.

Praticamente o número de clínicos gerais e especialistas do presente estudo eram o mesmo, diferentemente do estudo de Pinheiro e Delfino ${ }^{14}$ onde houve maior expressividade especialistas $65,69 \%$ e também do de Leôncio et al. ${ }^{18}$ com $70 \%$. Os tipos de pós-graduações odontológicas mais expressivas foram a Saúde da Família, seguida da Endodontia, ficando Odontopediatria, Dentística, Implantodontia, Odontologia do trabalho e Ortodontia com percentual de 3,3\% cada. Já os CD's que possuiam dois tipos de especialidade mostrou ser Saúde da Família e Ortodontia a mais expressiva com 6,7\%. E apenas 3,3\% da amostra revelou possuir 3 tipos sendo Periodontia, Endodontia e Saúde da Família. Em contrapartida o estudo de Granville-Garcia et al. ${ }^{21}$ mostrou ser a Ortodontia $(12,7 \%)$ a especialidade mais numerosa.

O fato da pós-graduação em Saúde da Família ter sido a mais observada é bastante considerável, devido ao setor de trabalho dos entrevistados, que são as Unidades Básicas de Saúde. Em relação a Cursos Em Emergência de Lesões Traumáticas uma minoria era possuidora, assim como no estudo de Pinheiro e Delfino $^{14}$ onde apenas $15,69 \%$ possuíam.

Dos entrevistados, 46,7\%, já recebeu pacientes com traumatismos dentários, dentre eles, predominando os casos de fraturas dentárias, seguido dos que relataram receber tanto fraturas como avulsão. No estudo de Lima et al. ${ }^{22}$ mostrou a avulsão sendo bem mais frequente com cerca de $(69,4 \%)$ das ocorrências.

Quanto às situações envolvendo trauma dental, $43,3 \%$ relataram que receberam e executaram o atendimento e $56,7 \%$ dos dentistas que receberam ou não paciente com TD diziam sentirem-se seguros para atender, o que no estudo de Kremer et al. ${ }^{3}$ em casos de trauma infantil a grande maioria $90,4 \%$ sentiam-se aptos.

Nos 10 casos clínicos propostos na parte II do questionário as respostas foram classificadas em: "certa" a melhor conduta que deveria ser adotada na situação explicitada e como resposta "errada" a conduta menos desejada.

Uma significativa maioria $(86,6 \%)$ afirmou a pulpotomia ser a medida a ser tomada nos casos de fratura esmalte-dentina-polpa com menos de $1 \mathrm{~h}$ em dentes com formação radicular incompleta. $\mathrm{O}$ resultado corrobora com o estudo de Ribeiro et al. ${ }^{23}$ em que a pulpotomia foi a técnica mais escolhida pelos dentistas para o tratamento de dentes com formação radicular incompleta $(\mathrm{n}=34,58,6 \%)$.

De acordo com Carvalho et al. ${ }^{24}$ a taxa de sucesso da pulpotomia nesses casos é alta, variando de $72,9 \%$ à $99,4 \%$, devido a grande capacidade reparadora da polpa mesmo que se apresente sintomatologia dolorosa e espontânea e rarefação óssea apical que são sinais e sintomas de alteração irreversível.

$\mathrm{Na}$ questão seguinte, em relação ao medicamento utilizado a grande maioria também declarou ser o Hidróxido de cálcio o material mais indicado nesse caso, sendo condizente com a terapêutica adotada pelos cirurgiões-dentistas anteriormente. Conforme Freires e Cavalcanti ${ }^{25}$, o efeito tecidual dos cimentos de hidróxido de cálcio quando aplicados sobre a polpa promove uma completa cicatrização dos tecidos pulpares e uma remineralização da dentina em forma de barreira tecidual. A sua utilização contribui para formação de pontes de dentina, expulsão de infiltrado bacteriano e recuperação tecidual.

Quanto aos casos de avulsão imediata de dente permanente, $86,6 \%$ dos dentistas indicariam à mãe da vítima por telefone que fosse realizado o reimplante e se o procedimento não pudesse ser realizado o dente poderia ser colocado em solução salina e ir para o consultório odontológico imediatamente, e já no consultório o procedimento seguinte adotado pela grande maioria $(93,3 \%)$ seria exame radiográfico, colocação de tala (contenção) e instruções sobre o controle da placa bacteriana e controle da dieta. Quanto ao tipo de tala que deveria ser usada, $73,3 \%$ indicam a semirrígida durante 2 semanas ou até que a mobilidade do dente se mostre reduzida, o que corrobora com Diangelis et al. ${ }^{26}$.

Em relação à prescrição de medicamentos neste mesmo caso citado, $33,3 \%$ dos dentistas disseram que era necessário apenas anti-inflamatório e analgésico, $23,3 \%$ que além desses, um antibiótico de espectro estreito e 43,3\% antiinflamatório, analgésico e antibiótico de espectro alargado, sendo a última a alternativa tida como certa, ou seja, o percentual de erro dessa questão foi de 56,6\%, consideravelmente elevado. Para Carmo et al. ${ }^{27} \mathrm{e}$ Guimarães et al. $^{28}$ o trauma dentário é uma das situações onde se usa mais frequentemente antibioticoterapia, além de reafirmarem a utilização de antibióticos para casos de avulsão dentária tanto para rizogênese completa ou incompleta assim como descreve a literatura. Para Andersson et al. ${ }^{29}$ a administração sistêmica de antibióticos tem como primeira escolha a Tetraciclina, porém, seu uso em paciente mais jovens deve ser cuidadoso pelo risco de descoloração permanente do elemento dentário, tendo como alternativa a Amoxicilina. De acordo com Trope ${ }^{30}$ a utilização de Amoxicilina no momento do reimplante e anterior ao tratamento endodôntico é eficiente na prevenção da invasão de bactérias à polpa necrosada e, consequentemente, a reabsorção inflamatória.

No caso de fratura coronária de esmalte e dentina, em que o paciente após $24 \mathrm{~h}$ reclamou de um pouco de dor a conduta em que deveria ser feita era o revestimento com hidróxido de cálcio aplicado à dentina exposta e em seguida restauração imediata foi prevalente com $66,6 \%$, o que se nota o conhecimento sobre as propriedades anti-inflamatórias do $\mathrm{Ca}(\mathrm{OH})^{2}$ e da necessidade de proteção do complexo dentina-polpa por boa parte dos dentistas.

Quanto à conduta frente um caso de fratura 
radicular horizontal no terço apical após $24 \mathrm{hrs}$, houve o maior número respostas consideradas erradas $(60 \%)$. O motivo talvez pela não maior especificação do tratamento a ser adotado nas alternativas, onde o tratamento endodôntico era o indicado. Segundo Diangelis et al. $^{26}$, se houver necrose pulpar, um tratamento endodôntico até a linha da fratura é indicado, por mais que seja relativamente infrequente e segundo Fagundes et al. ${ }^{31}$ e Mankar et al. ${ }^{32}$, em $25 \%$ dos casos é que vai ocorrer necrose pulpar do deslocamento da porção coronal, e esses mesmos autores acreditam que o uso de tala não seria muito apropriado uma vez que é quase anormal a ocorrência de mobilidade do segmento coronal. A cicatrização de forma espontânea da linha de fratura apical tem sido relatada em muitos casos, deste modo uma observação periódica tem sido defendida ${ }^{31,32}$. Em caso de elemento permanente avulsionado e mantido seco durante $7 \mathrm{~h}, 16,6 \%$ dos CD's não sabiam ou não tinham certeza de qual conduta tomar e 16,6\% indicavam a substituição dos dentes perdidos por prótese, sendo que a maioria respondeu de maneira correta as alternativas em que deveria se colocar o dente em uma solução de fluoreto (fluoreto de sódio 2,4\%), purificar o alvéolo com soro fisiológico, fazer o tratamento endodôntico antes ou depois de reimplantar, tala e antibioticoterapia. Segundo Kinirons et al. ${ }^{33}$, há uma crítica associação entre a cicatrização periodontal e o tempo em que o dente permanece fora do alvéolo em meio seco. Segundo Andersson et al. $^{29}$, antes do reimplante, é indicada a utilização da solução de fluoreto de sódio a $2 \%$ na superfície radicular por durante $20 \mathrm{~min}$, por atuar como afirmam Panzarini et al. ${ }^{34}$ reduzindo a reabsorção inflamatória e a evitando a ocorrência de áreas de anquilose e reabsorção por substituição.

A conduta em casos de reabsorção radicular interna foi a escolhido pela maioria, optando-se pelo tratamento endodôntico por meio de repetição de enchimento com hidróxido de cálcio antes do canal ser obturado definitivamente, o que está correto, mostrando mais uma vez uma das propriedades do $\mathrm{Ca}(\mathrm{OH})^{2}$ em ser remineralizante.

$\mathrm{Na}$ análise da relação entre a segurança em atender os pacientes com trauma dental e o perfil e conduta dos profissionais, embora nenhum dos itens tenha dados estatisticamente significantes, observou-se que $70 \%$ dos profissionais do sexo masculino se consideravam seguros para atender pacientes com trauma dental, enquanto que só $50 \%$ das mulheres também relataram.

A faixa etária dos $31-40$, de onde pertence a média de idade dos entrevistados (35,6 anos), foi a dos profissionais que mais se sentiram seguros em atender (75\%). Os anos de formação não influenciaram nesse aspecto, sendo que os que tinham até 5 anos de formação, $57,1 \%$, se sentiam seguros e os que possuíam mais de 5 anos representaram 56,2\%, assim como a presença ou não de Pós-graduação não pareceu influenciar na segurança no atendimento.

Dos que exercem atendimento apenas nas instituições públicas uma discreta maioria $(52,4 \%)$ relatou não se sentir seguro em atender pacientes com $\mathrm{TD}$, já os que exercem atendimentos em instituições públicas e privadas mostraram-se mais seguros, onde $77,8 \%$ relataram sentirem-se seguros. Quanto à conduta frente ao trauma, dos que nunca receberam casos do tipo $(68,8 \%)$ relataram sentir-se seguro caso houvesse uma ocorrência, e dos que já executaram atendimento de pacientes com traumatismo dentário nas Unidades de Saúde, 53,8\% dizia não se sentir apto para realizar tal fato, enquanto que os que realizaram encaminhamento declaram-se totalmente inseguros.

Algo discutível é que, mesmo realizando o atendimento, os cirurgiões-dentistas referiam não sentirem segurança suficiente para realizarem um correto atendimento aos pacientes que chegam com diferentes tipos de traumas dentários.

\section{CONCLUSÃO}

Os traumatismos dentoalveolares constituem uma situação relativamente frequente no cotidiano dos atendimentos clínicos das Unidades Básicas de Saúde de Patos-PB e observou-se que embora os CirurgiõesDentistas tenham demonstrado um conhecimento satisfatório em relação ao manejo do paciente com este tipo de lesão, uma quantidade significante afirmou não se sentir seguro para prestar o atendimento imediato a esses pacientes. Tal fato demonstra a necessidade de mais incentivo em cursos de formação na área para esses profissionais, o que viria a otimizar o atendimento ao paciente com traumatismo dentoalveolar.

\section{REFERÊNCIAS}

1. Duarte DA, Bonecker MS, Santanna GR, Suga SS. Caderno de Odontopediatria: Lesões traumáticas em dentes decíduos: Tratamento e Controle. São Paulo: Santos; 2001.

2. Piva F, Potter IG, Sari GT, Klein-Júnior CA, Coelho-De Souza FH. Atendimento de urgência frente ao traumatismo alvéolo dentário - relato de caso clínico. Rev Assoc Paul Cir Dent. 2013; 67(3):224-28.

3. Kremer JTM, Pereira LP, Marques FR, Portugal MEG, Bruzamolin, CD. Avaliação do conhecimento dos cirurgiões-dentistas sobre o manejo do trauma dental infantil. Rev Gestão \& Saúde. 2017;16(2):1-8.

4. Traebert J, Traiano ML, Armênio R, Barbieri DB, De Lacerda JT, Marcenes W. Knowledge of lay people and dentists in emergency management of dental trauma. Dent Traumatol. 2009;25(3):277-83.

5. Sb-Brasil 2010. Pesquisa Nacional de Saúde Bucal. Proposta de projeto técnico para consulta pública. Brasília; 2010.

6. Skaare $A B$, Jacobsen I. Primary tooth injuries in Norwegian children (1-8 years). Dent Traumatol. 2005;21(6):15-9.

7. Silva BM, Costa MMA, Almeida CEM, Maia AS, Carvalho OIC, Resende BG. Avaliação do conhecimento da abordagem de trauma dental pelos profissionais de creches. ConScientiae Saude. 2009; 8(1):65-73. 
8. Andreasen JO, Andreasen FM, Anderson L. Textbook and color atlas of traumatic injuries to the teeth. Hoboken: Wiley-Blackwell; 2013.

9. Torres Silva MC, Díaz YB, Díaz AMB, Figueredo EJ, García YR. Factores predisponentes de trauma dental en escolares del municipio Rafael Freyre. Ccm Holguín. 2017;21(3):798-808.

10. Pádua MN, Mendes FM, Benedetto MS, Volpi NA, Mello-Moura JCI, Bonini GAVC. Prevalência de lesões dentárias traumáticas em pré-escolares de escolas públicas e particulares. J Health Sci Inst. 2010;28(3):237-40.

11. Sanabe EM, Cavalcante BL, Coldebella RC, Abreue-Lima BCF. Urgências em traumatismos dentários: classificação, características e procedimentos. Rev Paul Pediatr 2009;27(4):447-51.

12. Wigen TI, Agnalt R, Jacobsen I. Intrusive luxationof permanent incisors in Norwegians aged 6-17 years:a retrospective study of treatment and outcome. Dent Traumatol. 2008;24(3):612-18.

13. Cortes MI, Marcenes W, Sheiham A. Impact of traumatic injuries to the permanent teeth on the oral health-related quality of life in 12-14-year-old children. Community Dent Oral Epidemiol. 2002;30(3):193-98.

14. Pinheiro SAA, Delfino CS. Conhecimento do cirurgião-dentista sobre trauma dentário. Rev cir traumatol buco-maxilo-fac. 2014;14(1):83-90.

15. Granville-Garcia AF, Balduíno Jr JB, Ferreira JMS, Menezes VA, Fontes LBC, Cavalcanti AL. Conhecimento do cirurgião-dentista sobre avulsão dental no Programa de Saúde da Família de Campina Grande- PB/Brasil. Odonto. 2009; 17(33):35-41

16. Costa LED, Queiroz FS, Nóbrega CBC, Leite MS, Nóbrega WFS, Almeida ER. Trauma dentário na infância: avaliação da conduta dos educadores de creches públicas de Patos-PB. Rev Odontol UNESP. 2014;43(6):402-8.

17. Hu LW, Prisco CRD, Bombana AC. Knowledge of Brazilian general dentists and endodontists about the emergency management of dentoalveolar trauma. Dent Traumatol. 2006;22(3):113-17.

18. Leôncio LL, Batista EPC, Nóbrega CBC, Costa LED. Diagnóstico e encaminhamento de pacientes com doenças bucais no serviço público de saúde de Patos-PB: atuação do cirurgião-dentista na referência e contra referência. Arq Odontol. 2015;51(4):210-15.

19. Antunes Drusila P, Antunes Débora P, Chaoubat A, Paula MVQ de, Salgado IO, Coelho, LGC. O conhecimento de cirurgiões-dentistas sobre condutas clínicas nas avulsões e reimplantes dentários: estudo piloto. HU Rev. 2012;38(3,4):135-41.

20. França RI, Traebert J, De Lacerda JT. Brazilian dentists' knowledge regarding immediate treatment of traumatic dental injuries. Dent Traumatol. 2007;23(5):287-90.

21. Granville-Garcia AF, Menezes VA, Lopes I, Araujo OS, Fontes LBC, Cavalcanti AL. Conduta terapêutica dos cirurgiões-dentistas em relação aos traumatismos dentários. Arq Ciênc Saúde Unipar. 2008;12(3):239-47.

22. Lima DC, Pereira AA, Swerts AA, Fernandes LA. Conduta dos cirurgiões-dentistas de Alfenas/MG frente ao tratamento emergencial de pacientes com avulsão dentária. Arq Odontol. 2013;49(4):169-76.

23. Ribeiro ILA, Melo RTC, Trigueiro DA, Ferreira GS. Conduta clínica de cirurgiões-dentistas de João Pessoa-PB no tratamento endodôntico de dentes com rizogênese incompleta Rev. Odontol Univ Cid São Paulo. 2014;26(3):212-18.

24. Carvalho C, Freire LG, Nakamura V, Gavini G. Possibilidades terapêuticas no tratamento de dentes jovens portadores de polpa viva: revisão de literatura. Rev Ciênc Saúde. 2012;14(1):40-52.

25. Freires IA, Cavalcanti YW. Proteção do complexo dentinipulpar: indicações, técnicas e materiais para uma boa prática clínica. Rev Bras Pesq Saúde. 2011;13(4):69-80.

26. Diangelis AJ, Andreasen JO, Ebeleseder KA, Kenny DJ, Trope M, Sigurdsson A et al. International Association of Dental Traumatology guidelines for the management of traumatic dental injuries: 1 . Fractures and luxations of permanent teeth. Dent Traumatol. 2012;28(1)2-12.

27. Pereira AC, Silveira VAS, Rosa LEB, Rocha RF. Prescrição medicamentosa em odontopediatria. Rev odontol UNESP. 2009;38(4):256-62.

28. Guimarães S, Moura D, Silva PS. Terapêutica Medicamentosa e Suas Bases Farmacológicas. 5.ed. Porto: Porto Editora, 2006.

29. Andersson L, Andreasen JO, Day P, Heithersay G, Trope M, Diangelis AJ et al. International Association of Dental Traumatology guidelines for the management of traumatic dental injuries: 2 . Avulsion of permanent teeth. Dent Traumatol. 2012;28(2):88-96.

30. Trope, M. Clinical management of the avulsed tooth: Present strategies and future directions. Dent Traumatol. 2002;18(1):1-11.

31. Fagundes Ddos, de Mendonça IL, de Albuquerque MT, Inojosa Ide F. Spontaneous healing responses detected by cone-beam computed tomography of horizontal root fractures: a report of two cases. Dent Traumatol. 2014;30(6):484-87.

32. Mankar N, Jogad N, Chute A, Patil S, Gade V, Mohkar S. Management of horizontal root fracture: two case reports. IOSR J Dent Med Sci. 2014;13(4):1-4.

33. Kinirons MJ, Gregg TA, Welbury RR, Cole BO. Variations in the presenting andt treatment features in reimplant permanent incisors in children and their effect on the prevalence of root resorption. Br Dent J. 2000;189(5):263-66.

34. Panzarini SR, Gulineli JL, Poi WR, Sonoda CK, Pedrini D, Brandini DA. Treatment of root surface in delayed tooth replantation: a review of literature. Dent Traumat. 2008;24(3):277-82. 
http://dx.doi.org/10.21270/archi.v8i7.3803

\section{CONFLITO DE INTERESSES}

Os autores declaram não haver conflitos de interesse.

AUTOR PARA CORRESPONDENCIA

Faldryene de Sousa Queiroz

falqueiroz@hotmail.com

Submetido em 12/11/2018

Aceito em 12/03/2019 